\title{
BMJ Open Prevalence and incidence of dry eye in the USA: a systematic review protocol
}

\author{
Paul McCann (D , ${ }^{1}$ Alison G Abraham, ${ }^{2}$ Darren G Gregory, ${ }^{1}$ Scott Hauswirth, ${ }^{1}$ \\ Cristos Ifantides, ${ }^{1}$ Su-Hsun Liu, ${ }^{1}$ Ian J Saldanha, ${ }^{3}$ Tianjing Li ${ }^{1}$
}

To cite: McCann P, Abraham AG, Gregory DG, et al. Prevalence and incidence of dry eye in the USA: a systematic review protocol. BMJ Open 2021;11:e056203. doi:10.1136/ bmjopen-2021-056203

- Prepublication history and additional supplemental material for this paper are available online. To view these files, please visit the journal online (http://dx.doi.org/10.1136/ bmjopen-2021-056203).

Received 05 August 2021 Accepted 05 November 2021

Check for updates

(c) Author(s) (or their employer(s)) 2021. Re-use permitted under CC BY-NC. No commercial re-use. See rights and permissions. Published by BMJ.

${ }^{1}$ Department of Ophthalmology, University of Colorado Anschutz Medical Campus, Aurora, Colorado, USA ${ }^{2}$ Department of Epidemiology, Colorado School of Public Health, and Department of Ophthalmology, University of Colorado - Anschutz Medical Campus, Aurora, Colorado, USA ${ }^{3}$ Health Services, Policy and Practice, Brown University School of Public Health, Providence, Rhode Island, USA

Correspondence to

Dr Tianjing Li;

TIANJING.LI@CUANSCHUTZ.EDU

\section{ABSTRACT}

Introduction Dry eye is a multifactorial chronic condition characterised by tear film insufficiency and instability, and ocular symptoms including foreign body sensation, itching, irritation, soreness and visual disturbance. The prevalence and incidence of dry eye are major determinants of the magnitude of economic and societal costs of the disease. This protocol proposes a systematic review and metaanalysis of the prevalence and incidence of dry eye in the USA.

Methods and analysis Working with an information specialist, we will develop search strategies for Ovid Medline and Embase for population-based cross-sectional and cohort studies involving US-based populations that report the prevalence and/or incidence of dry eye. We will include studies involving persons of all ages from 1 January 2010 to the current date with no language restrictions. We will also hand-search references of included studies, dry eye epidemiology-related systematic reviews, clinical practice guidelines and literature provided by agencies and organisations. Two investigators will independently screen the titles and abstracts, and then full-text reports to determine eligibility. One investigator will extract study data and perform risk of bias assessments using tools designed specifically for prevalence and incidence studies. A second investigator will verify all extracted study data and risk of bias assessments. We will assess heterogeneity, qualitatively and quantitatively. When appropriate, we will meta-analyse prevalence and incidence estimates.

Ethics and dissemination This review does not require approval by an ethics committee because it will use published studies. We will publish our results in a peerreviewed journal and present at relevant conferences. PROSPERO registration number CRD42021256934.

\section{INTRODUCTION}

Dry eye disease (DED) is defined by the Tear Film and Ocular Surface Society (TFOS) Dry Eye Workshop II (DEWS-II) as 'a multifactorial disease of the ocular surface characterised by a loss of homeostasis of the tear film, and accompanied by ocular symptoms, in which tear film instability and hyperosmolarity, ocular surface inflammation and damage, and neurosensory abnormalities play etiological roles. ${ }^{1}$ Because there is no gold standard diagnostic test for DED, the term 'dry eye' is used to describe various presentations of
Strengths and limitations of this study

We aim to overcome limitations in previous reviews of dry eye epidemiology reports.

- We will use contemporaneous data and comprehensive methods to enhance transparency and reproducibility.

- We anticipate high levels of heterogeneity in prevalence and incidence estimates; however, we aim to explore the reasons for heterogeneity.

ocular discomfort and tear film abnormalities. Dry eye is frequently referred to as DED once it is clinically diagnosed. ${ }^{2}$

Irrespective of a clinical diagnosis of DED, dry eye causes considerable burden to patients and society. Patient burden includes decreased quality of life due to symptoms, such as foreign body sensation, itching, irritation, soreness and visual disturbance, which interfere with reading, driving, and work productivity, and cause physical and emotional distress. ${ }^{3-5}$ Burdens to society include direct economic costs (eg, healthcare professional visits, treatment costs $){ }^{6}$ non-direct economic costs (eg, work productivity $\operatorname{loss})^{7}$ and intangible personal costs (eg, impaired social, emotional and physical functioning) ${ }^{8}{ }^{9}$ In 2011, the estimated direct economic cost to the US healthcare system for DED therapy was US $\$ 3.8$ billion per year and the estimated total societal cost in the USA was US $\$ 55.4$ billion per year. ${ }^{6}$ Comparative analyses have demonstrated that DED-related costs in the USA are broadly comparable with other countries. ${ }^{10}$ However, in the USA, personal costs may be higher because treatments, such as ocular lubricants, may not be adequately covered by health insurance, and drug costs tend to be higher in the USA. ${ }^{611}$ With introduction of newer and more costly therapies, an even larger societal and personal economic burden of dry eye can be expected. ${ }^{12-14}$ Furthermore, despite being a significant public health problem, dry eye remains underdiagnosed, highlighting the 
likelihood that there is a significant undiagnosed burden of disease. ${ }^{2} 1516$

In 2017, a comprehensive epidemiology report by the TFOS DEWS-II ('TFOS epidemiology report') reviewed population-based studies that enrolled at least 500 participants to estimate the prevalence and incidence of dry eye stratified by definition of disease, age, sex and worldwide geographical region. ${ }^{17}$ The findings of the TFOS report showed that, globally, the prevalence of dry eye ranged from $5 \%$ to $50 \%$ with various definitions of DED. However, in dry eye, as well as in other ophthalmic diseases, applying differing definitions of disease to epidemiological datasets can result in widely varying estimates of prevalence. $^{18}$

In addition to disease definition, various factors may contribute to differences in prevalence of dry eye. ${ }^{17}$ The prevalence has been reported to increase with age, especially in women. ${ }^{151719}$ To our knowledge, few studies have reported prevalence in people younger than 21 years old, and none were in US-based populations. ${ }^{19-21}$ This lack of data is problematic because young people are also at risk of dry eye due to generally longer screen time (eg, video monitors, digital tablets), and contact lens wear. ${ }^{20}$ The TFOS report found no clear pattern of dry eye associated with latitude, globally. ${ }^{17}$ However, in the USA, there is indirect evidence of an association with latitude, with higher prevalence of dry eye reported in southern regions of the country. ${ }^{215}$ Furthermore, other geo-environmental factors, such as higher atmospheric pressure, air pollution, humidity and wind speed, have all been shown to be risk factors for dry eye. ${ }^{22}$ As the USA comprises an expansive land mass with great variation in climate across latitudinal and topographical regions, and given that climatic factors are influential risk factors for dry eye, it is important to consider these factors when estimating prevalence and incidence of dry eye.

The literature search for the TFOS epidemiology report covered a 10-year period from 2005 to 2015 (last updated on 17 September 2015). However, it is unclear whether the TFOS epidemiology report strictly followed critical steps in the systematic review process, such as protocol development, risk of bias assessment and appropriate meta-analysis. ${ }^{17}$ Furthermore, the TFOS epidemiology report is now relatively dated because more dry eye-related epidemiological studies have been performed in the USA since its publication. ${ }^{23}$

Systematic reviews of dry eye-related epidemiology have been published for other populations and global regions but, ${ }^{2425}$ to our knowledge, there are no existing systematic reviews of dry eye epidemiology within the USA. As the prevalence and incidence of dry eye are major determinants of the magnitude of the personal, societal and economic costs of the disease, examining these epidemiological indices can help health policymakers estimate the burden of dry eye in the USA and consequently allocate resources to risk mitigation and treatment as needed.

\section{Primary objective}

The primary objective of this systematic review and metaanalysis is to summarise the prevalence and incidence of dry eye in persons of all ages in the USA.

\section{Secondary objectives}

1. Estimate the effect of disease definition, age group, sex, US region and geoenvironmental factors on prevalence and incidence of dry eye in the USA by using metaregression methods.

2. Assess heterogeneity in the prevalence and incidence of dry eye within the USA and factors potentially explaining the heterogeneity.

3. Report epidemiological factors associated with dry eye.

\section{METHODS AND ANALYSIS}

We have registered for this systematic review protocol with the PROSPERO international register for systematic reviews (CRD42021256934) and we report it in accordance with the Preferred Reporting Items for Systematic Reviews and Meta-Analysis Protocols 2015 statement (see online supplemental file 1). We will conduct and report the review with guidance from the Joanna Briggs Institute Manual for Evidence Synthesis, ${ }^{26}$ the Cochrane Handbook for Systematic Reviews of Interventions, ${ }^{27}$ the Meta-analysis of Observational Studies in Epidemiology guidelines, ${ }^{28}$ the Guidelines for Accurate and Transparent Health Estimates Reporting statement ${ }^{29}$ and a meta-epidemiological study on the assessment of prevalence study quality by Migliavaca $e t a l .{ }^{30}$

\section{Patient and public involvement \\ No patient involved.}

\section{Criteria for considering studies for this review}

We used the populations, context and condition framework for the systematic review of prevalence and incidence to formulate the eligibility criteria. ${ }^{31}$

Population and context

We will investigate the prevalence and incidence of dry eye in the US population (ie, the target population). Prevalence is the proportion of the population with dry eye at a given time (point or period of time). Cumulative incidence is the proportion of persons in the at-risk population who develop a new diagnosis of dry eye during a given follow-up period. Incidence rate is the number of new cases of dry eye divided by the observed person-time during a given observation period. We aim to explore the influence of demographic factors (eg, age, sex), environmental exposures (eg, air pollution, screen time), meteorological exposures (eg, temperature, wind speed, relative humidity, atmospheric pressure) and underlying risk factors of disease (eg, comorbidities, topical and systemic medications) on these epidemiological indices. Our source populations will be from studies conducted within the USA and studies conducted outside the USA are not eligible. However, the target population may be 
broadened to Continental North American populations if there is a sparsity of US-based studies (ie, less than two US-based studies) although this is not expected.

\section{Condition}

We will use definitions of dry eye outlined in the included primary studies. We will aim to consolidate similar case definitions across studies into homogenous definitions when appropriate. In the TFOS report, case definitions of DED included: (1) Women's Health Study (WHS) criteria (ie, self-reported physician diagnosis and/or selfreported 'constant' or 'often' symptoms), ${ }^{15}$ (2) dry eye symptoms when signs were not measured (eg, measured by the Ocular Surface Disease Index), (3) dry eye clinical signs when symptoms were not measured (eg, tear break up time), (4) a combination of dry eye signs and symptoms (distinct from WHS criteria) and (5) Meibomian gland dysfunction. ${ }^{17}$ We will also include dry eye definitions based on relevant International Classification of Disease codes.

\section{Types of studies}

We will include population-based observational studies (ie, cross-sectional studies and cohort studies) that reported prevalence or incidence of dry eye in the USA. We will not exclude studies based on characteristics such as sampling frame or sampling methods, but these will be assessed as part of the risk-of-bias assessment of included studies. We will exclude case reports, case series, case-control studies and interventional studies. We will exclude population-based studies with fewer than 73 total participants because estimates from samples with less than 73 participants would produce $95 \%$ CIs greater than \pm 0.05 when the anticipated minimum population proportion is estimated to be $0.05 .{ }^{32}$ However, if we find studies on specific population subgroups (eg, native Americans) that have fewer than 73 total participants we will consider them for inclusion.

\section{Search methods for identification of studies \\ Electronic searches}

Working with an information specialist, we will develop search strategies for Ovid Medline, and Embase for population-based studies that report the prevalence and/ or incidence of dry eye. We will include studies involving persons with all ages from 1 January 2010 to the current date with no language restrictions. The search strategy will include text word as well as controlled vocabulary (eg, medical subject headings, Emtree) terms for epidemiological concepts, such as "epidemiology", "prevalence", "incidence" and "burden of disease", combined with dry eye-related concepts, such as "dry eye syndromes" (see online supplemental file 2).

\section{Other sources}

We will hand-search references of included studies, dry eye epidemiology-related systematic reviews and clinical practice guidelines for additional studies. Conference abstracts will be searched as part of our electronic search of Embase. We will search literature provided by agencies including the WHO. We will contact study authors for complete data to calculate prevalence and/or incidence when required.

\section{Data collection and analysis}

\section{Selection of studies}

We will remove duplicate records and import the search results into Covidence, a web-based review management software. ${ }^{33}$ Then, two investigators will independently screen each title and abstract. Investigators will classify each record as 'yes' (relevant), 'maybe' (possibly relevant) and 'no' (not relevant) for further full-text review. During title/abstract screening, studies that meet the eligibility criteria for population, context and condition will be included for full text screening.

We will retrieve the full-text articles for records considered 'relevant' or 'possibly relevant'. Then, two investigators will independently screen the full-text articles for eligibility and classify articles as 'to be included' or 'to be excluded'. If there are questions regarding the eligibility of a given study, we will contact its authors to obtain additional information. If the authors do not respond to three emails within 4 weeks, we will use information available from study reports to determine eligibility.

During the screening process, we will exclude but tag studies of non-US-based populations that otherwise meet the eligibility criteria. This will prove useful should the population eligibility criteria be broadened (ie, Continental North American populations) due to sparsity of US-based studies.

We will review studies in languages other than English that reach full text review based on their title and abstract following translation by Google Translate when possible. We will report reasons for exclusion of full texts in an 'Excluded Studies' table. We will classify studies that meet eligibility criteria but have not yet been completed or have not published full text reports within 2 years of completion as 'ongoing'. We will resolve discrepancies regarding the classification of the studies by discussion and, where needed, adjudication by a third investigator.

\section{Data extraction and management}

One investigator will extract all relevant study characteristics and other information from included studies into a data collection form using a platform such as the Systematic Review Data Repository Plus. An independent investigator will verify the information for accuracy. ${ }^{34} \mathrm{We}$ will resolve discrepancies by consensus or, if consensus cannot be reached, by adjudication by a third investigator. Where available, we will extract the following data: article information (first author's name, year of publication, country and region where the study was conducted), study design, source population, study population, participant inclusion and exclusion criteria, sampling method, sample size at baseline, index date, dates of follow-up, follow-up period, region(s) where the participants were recruited, case definition(s), participant characteristics 
(eg, age, sex), prevalence, prevalence period, cumulative incidence, incidence rate and measures of precision. We will extract from each study, all factors included in association analyses (eg, age and sex). We will extract estimates (eg, relative risk) and their precisions for unadjusted and adjusted factors associated with disease. We will record which covariates were included in the multivariable adjusted models of disease association.

\section{Assessment of risk of bias in included studies}

One review author will assess the risk of bias in each included study using specific risk of bias tools for prevalence and incidence studies. Another investigator will independently verify the information. ${ }^{34}$ Any conflicts will be resolved by discussion or by adjudication by a third investigator. We will provide tool guides a priori for consistent and transparent use of each tool among investigators.

For prevalence studies, we will use the tool proposed by Hoy et $a l^{35}$ Items $1-4$ of the tool assess the external validity of the study (items 1 and 2 assess sampling bias, and items 3 and 4 assess non-response bias). For item 1 , we will address the extent to which the study population represents the general US population with respect to factors that influence prevalence and incidence of dry eye. Items 5-10 assess internal validity (items 5-9 assess ascertainment bias, and item 10 assesses bias related to the analysis). The study is rated as 'high' or 'low' risk of bias for each of the 10 items; there is no 'unclear' option. Once all 10 items are rated, we will evaluate the overall risk of bias in the summary assessment. The summary assessment is a subjective judgement and is not calculated as an overall sum of the items. There are three options for the summary assessment: high, 'moderate', and low risk of bias.

For incidence studies, we will use the Joanna Briggs Institute Critical Appraisal Checklist for Cohort Studies. ${ }^{36}$ The checklist has 11 items, and each item has 'yes', 'no', 'unclear', and 'not applicable' options. There is an additional overall appraisal item with 'include', 'exclude' and 'seek further info' options, and a comment section for the 'reason of exclusion'. We will not exclude studies from the systematic review based on the 'exclude' response in the overall appraisal item, but we will interpret this response as 'high risk of bias'. We will consider excluding studies from meta-analysis based on an 'exclude' response in the overall appraisal item (ie, high risk of bias).

\section{Data synthesis}

We will summarise from each study, sample characteristics and prevalence and incidence data with precision estimates, in structured tables. ${ }^{37}$ We will also present all reported potential risk factors for dry eye including their definitions (eg, age grouping) and effect estimates for each potential risk factor, including specific risk factors such as geo-environmental factors and screen time when data are available. We will document prevalence and incidence of dry eye severity using previously defined classifications when reported in the primary studies. ${ }^{38}{ }^{39}$ All data will be stratified by case definition whenever feasible.

\section{Investigation of heterogeneity}

We will qualitatively investigate sources of heterogeneity of the data by assessing risk of bias and other aspects of the design of each study (methodological heterogeneity) and examining the characteristics of the populations (clinical heterogeneity) in each study, including age, sex, case definition and sociodemographic profiles. We will display the estimates and their uncertainty from each study in forest plots (separately for prevalence and incidence). We will quantitatively assess statistical heterogeneity by calculating the amount of heterogeneity $\left(\tau^{2}\right)$ and the contribution of heterogeneity to the total variability across studies $\left(I^{2}\right){ }^{40}$

\section{Meta-analyses}

When appropriate, we will conduct meta-analyses of prevalence and incidence estimates. We will combine data if the study estimates have acceptable heterogeneity, both qualitatively and quantitatively. If a study uses more than one case definition and reports several prevalence and incidence estimates, we will stratify the estimates by case definition and analyse them in separate subgroup metaanalyses. We will use our clinical expertise and the literature to judge which case definitions are compatible for pooling in subgroup meta-analyses. We will also consider stratifying meta-analyses by levels of risk of bias. We will consider meta-analysis of measures of association for common risk factor covariates across studies. Whether or not we conduct meta-analyses, we will qualitatively summarise the findings across studies in a summary of findings table.

We will meta-analyse prevalence and cumulative incidence proportions using separate random-intercept regression models with a logistic link function via the exact likelihood method. We will combine incidence rate using a random-intercept regression model. Both models and can be fitted in the generalised linear mixed model modules available in many popular statistical packages such as SAS, R and Stata. ${ }^{41}$

\section{Metaregression}

If there are sufficient risk factor data within-sample (ie, from the primary studies) and out-of-sample (eg, from census-derived demographic data, governmental agency derived geo-environmental data), we will consider conducting a Bayesian meta-regression with integrative systems modelling using DisMod-MR software. ${ }^{42}$ This will allow us to extrapolate nationwide prevalence and incidence estimates captured in the primary studies and stratify prevalence and incidence by factors such as age, sex, US region and geo-environmental factors. ${ }^{42-44}$ Integrative systems modelling potentially addresses some of the notable challenges faced in this meta-analysis including, (1) diverse case definitions, (2) variation in environmental and climatic exposures within the country 
and (3) a lack of standardised age stratification), which may improve compatibility for pooling of data. We will consult with statisticians and integrative systems modelling experts to decide on the most appropriate statistical approach.

\section{DISCUSSION}

DED is a chronic symptomatic condition that is costly to society, reduces quality of life and is among the leading reasons for presentation to eye care services worldwide. For this reason, the WHO has emphasised that dry eye must not be overlooked when addressing global eye care needs. ${ }^{45}$ With demographic ageing, ${ }^{46}$ lifestyle changes ${ }^{24}$ climate changes ${ }^{21522}$ and the introduction of newer and more costly therapies, ${ }^{13}$ dry eye-related economic costs to the US society can be expected to increase considerably. Hence, contemporaneous burden of disease estimates are necessary to enable health policy-makers and research funding bodies to make decisions regarding public health interventions and adequate resource allocation.

Our systematic review and meta-analysis will overcome some of the limitations in previous reviews of dry eye epidemiology reports as we will use contemporaneous data and comprehensive methods to enhance transparency and reproducibility. However, we do anticipate challenges and limitations in our study. An important limitation will be the anticipated high levels of heterogeneity in prevalence and incidence estimates. But this will provide the opportunity to explore and report the reasons for heterogeneity such as clinical and methodological variations. Another limitation is that we will search only published literature and we acknowledge the potential of publication bias. Despite potential limitations, the information gathered from this study is likely to be widely used in the USA and in comparable settings by patients, physicians, health policy-makers, researchers and custodians to obtain and allocate funds and other resources to target the prevention and treatment of dry eye.

\section{ETHICS AND DISSEMINATION}

This review does not require the approval of an ethics committee because it will use previously published studies. We will publish our results in a peer-reviewed journal and present at relevant conferences

Acknowledgements We would like to acknowledge the contribution of Kristen Desanto, our information specialist, who assisted us with developing the search strategy for electronic databases. We would also like to acknowledge and thank Dr Abraham Flaxman (University of Washington) for reviewing and consulting on our proposed meta-regression methods.

Contributors PMM: concept, design, drafting, final submission. AGA: statistical and methodological design, drafting. DGG, SH and Cl: design, drafting. S-HL: methodological design, drafting. IS: methodological design, drafting, final submission. TL: concept, design, drafting, final submission, guarantor.

Funding This work was supported by National Eye Institute, National Institutes of Health, grant number UG1EY020522. The funding body had no role in developing the protocol.

Competing interests None declared.
Patient consent for publication Not applicable.

Provenance and peer review Not commissioned; externally peer reviewed.

Supplemental material This content has been supplied by the author(s). It has not been vetted by BMJ Publishing Group Limited (BMJ) and may not have been peer-reviewed. Any opinions or recommendations discussed are solely those of the author(s) and are not endorsed by BMJ. BMJ disclaims all liability and responsibility arising from any reliance placed on the content. Where the content includes any translated material, BMJ does not warrant the accuracy and reliability of the translations (including but not limited to local regulations, clinical guidelines, terminology, drug names and drug dosages), and is not responsible for any error and/or omissions arising from translation and adaptation or otherwise.

Open access This is an open access article distributed in accordance with the Creative Commons Attribution Non Commercial (CC BY-NC 4.0) license, which permits others to distribute, remix, adapt, build upon this work non-commercially, and license their derivative works on different terms, provided the original work is properly cited, appropriate credit is given, any changes made indicated, and the use is non-commercial. See: http://creativecommons.org/licenses/by-nc/4.0/.

ORCID iD

Paul McCann http://orcid.org/0000-0002-6950-4288

\section{REFERENCES}

1 Craig JP, Nichols KK, Akpek EK, et al. TFOS DEWS II definition and classification report. Ocul Surf 2017;15:276-83.

2 Farrand KF, Fridman M, Stillman Ipek Özer, et al. Prevalence of diagnosed dry eye disease in the United States among adults aged 18 years and older. Am J Ophthalmol 2017;182:90-8.

3 Schiffman RM, Walt JG, Jacobsen G, et al. Utility assessment among patients with dry eye disease. Ophthalmology 2003;110:1412-9.

4 Miljanović B, Dana R, Sullivan DA, et al. Impact of dry eye syndrome on vision-related quality of life. Am J Ophthalmol 2007;143:409-15.

5 Galor A, Feuer W, Lee DJ, et al. Depression, post-traumatic stress disorder, and dry eye syndrome: a study utilizing the National United States Veterans Affairs Administrative Database. Am J Ophthalmol 2012;154:340-6.

$6 \mathrm{Yu} \mathrm{J}$, Asche CV, Fairchild CJ. The economic burden of dry eye disease in the United States: a decision tree analysis. Cornea 2011;30:379-87.

7 Nichols KK, Bacharach J, Holland E, et al. Impact of dry eye disease on work productivity, and patients' satisfaction with over-the-counter dry eye treatments. Invest Ophthalmol Vis Sci 2016;57:2975-82.

8 Mertzanis P, Abetz L, Rajagopalan K. The relative burden of dry eye in patients' lives: comparisons to a US normative sample. Invest Ophthalmol Vis Sci 2005;46:46-50.

9 Reddy P, Grad O, Rajagopalan K. The economic burden of dry eye: a conceptual framework and preliminary assessment. Cornea 2004;23:751-61.

10 McDonald M, Patel DA, Keith MS, et al. Economic and humanistic burden of dry eye disease in Europe, North America, and Asia: a systematic literature review. Ocul Surf 2016;14:144-67.

11 Mulcahy AW, Whaley CM, Gizaw M. International prescription drug price comparisons: current empirical estimates and comparisons with previous studies. Santa Monica, CA: RAND Corporation PP, 2021.

12 Chen EM, Kombo N, Teng CC, et al. Ophthalmic medication expenditures and out-of-pocket spending: an analysis of United States prescriptions from 2007 through 2016. Ophthalmology 2020;127:1292-302.

13 Holland EJ, Luchs J, Karpecki PM, et al. Lifitegrast for the treatment of dry eye disease: results of a phase III, randomized, double-masked, placebo-controlled trial (OPUS-3). Ophthalmology 2017;124:53-60.

14 Pan Q, Angelina A, Marrone M. Autologous serum eye drops for dry eye. Cochrane Database Syst Rev 2017;2:CD009327.

15 Schaumberg DA, Sullivan DA, Buring JE, et al. Prevalence of dry eye syndrome among US women. Am J Ophthalmol 2003;136:318-26.

16 Shimmura S, Shimazaki J, Tsubota K. Results of a population-based questionnaire on the symptoms and lifestyles associated with dry eye. Cornea 1999;18:408-11.

17 Stapleton F, Alves M, Bunya VY, et al. TFOS DEWS II epidemiology report. Ocul Surf 2017;15:334-65.

18 Wolfs RCW, Borger PH, Ramrattan RS. Changing views on openangle glaucoma: definitions and prevalences - the Rotterdam study. Investig Ophthalmol Vis Sci 2000;41:3309-21.

19 Paulsen AJ, Cruickshanks KJ, Fischer ME, et al. Dry eye in the beaver dam offspring study: prevalence, risk factors, and healthrelated quality of life. Am J Ophthalmol 2014;157:799-806. 
20 Uchino M, Dogru M, Uchino Y, et al. Japan Ministry of health study on prevalence of dry eye disease among Japanese high school students. Am J Ophthalmol 2008;146:925-9.

21 Tan LL, Morgan P, Cai ZQ, et al. Prevalence of and risk factors for symptomatic dry eye disease in Singapore. Clin Exp Optom 2015;98:45-53.

22 Galor A, Kumar N, Feuer W, et al. Environmental factors affect the risk of dry eye syndrome in a United States veteran population. Ophthalmology 2014;121:972-3.

23 Dana R, Bradley JL, Guerin A, et al. Estimated prevalence and incidence of dry eye disease based on coding analysis of a large, all-age United States health care system. Am J Ophthalmol 2019;202:47-54.

24 Courtin R, Pereira B, Naughton G, et al. Prevalence of dry eye disease in visual display terminal workers: a systematic review and meta-analysis. BMJ Open 2016;6:e009675.

25 Liu N-N, Liu L, Li J, et al. Prevalence of and risk factors for dry eye symptom in mainland China: a systematic review and meta-analysis. J Ophthalmol 2014;2014:748654.

26 Aromataris E, Munn Z. JBI manual for evidence synthesis. JBI, 2020

27 Higgins J, Thomas J, Chandler J. Cochrane handbook for systematic reviews of interventions version 6.2 (updated February 2021), 2021.

28 Stroup DF, Berlin JA, Morton SC, et al. Meta-analysis of observational studies in epidemiology: a proposal for reporting. Meta-analysis of Observational Studies in Epidemiology (MOOSE) group. JAMA 2000;283:2008-201.

29 Stevens GA, Alkema L, Black RE, et al. Guidelines for accurate and transparent health estimates reporting: the gather statement. Lancet 2016;388:e19-23.

30 Migliavaca CB, Stein C, Colpani V, et al. Quality assessment of prevalence studies: a systematic review. J Clin Epidemiol 2020;127:59-68.

31 Munn Z, Moola S, Lisy K, et al. Methodological guidance for systematic reviews of observational epidemiological studies reporting prevalence and cumulative incidence data. Int J Evid Based Healthc 2015;13:147-53.

32 Lemeshow S, Hosmer Jr DW, Klar J. Adequacy of sample size in health studies. World Health Organization, 1990.

33 Veritas Health Innovation Melbourne Australia. Covidence systematic review software.
34 Jian-Yu E, Saldanha IJ, Canner J, et al. Adjudication rather than experience of data abstraction matters more in reducing errors in abstracting data in systematic reviews. Res Synth Methods 2020;11:354-62.

35 Hoy D, Brooks P, Woolf A, et al. Assessing risk of bias in prevalence studies: modification of an existing tool and evidence of interrater agreement. J Clin Epidemiol 2012;65:934-9.

36 Moola S, Munn Z, Tufanaru C. Chapter 7: Systematic reviews of etiology and risk. In: Aromataris E, Munn Z, eds. Joanna briggs institute reviewer's manual. The Joanna Briggs Institute, 2017.

37 McKenzie JE, Brennan SE. Synthesizing and presenting findings using other methods. In: Higgins J, Thomas J, Chandler J, et al, eds. Cochrane handbook for systematic reviews of interventions version 6.2 (updated February 2021), 2021.

38 The definition and classification of dry eye disease: report of the definition and classification Subcommittee of the International dry eye workshop. Ocul Surf 2007;5:75-92.

39 Wolffsohn JS, Arita R, Chalmers R, et al. TFOS DEWS II diagnostic methodology report. Ocul Surf 2017;15:539-74.

40 Rücker G, Schwarzer G, Carpenter JR, et al. Undue reliance on I(2) in assessing heterogeneity may mislead. BMC Med Res Methodol 2008;8:79.

41 Stijnen T, Schmid CH, Law M. Exact likelihood methods for group-based summaries. In: Schmid CH, Stijnen T, White IR, eds. Handbook of meta-analysis. CRC Press, 2021: 65-89.

42 Flaxman AD, Vos T, Murray CJ. An integrative metaregression framework for descriptive epidemiology. University of Washington Press, 2015.

43 Flaxman AD, Wittenborn JS, Robalik T, et al. Prevalence of visual acuity loss or blindness in the US: a Bayesian meta-analysis. JAMA Ophthalmol 2021;139:717-23.

44 Bourne R, Steinmetz JD, Flaxman S, et al. Trends in prevalence of blindness and distance and near vision impairment over 30 years: an analysis for the global burden of disease study. Lancet Glob Health 2021;9:e130-43.

45 World Health Organization. World report on vision. Geneva: World Health Organization, 2019.

46 Christensen K, Doblhammer G, Rau R, et al. Ageing populations: the challenges ahead. Lancet 2009;374:1196-208. 